\title{
Office of Logistics and Acquisition Operations
}

National Cancer Institute

\section{Source}

National Cancer Institute. Office of Logistics and Acquisition Operations. NCI Thesaurus.

Code C82629.

An office of the National Institutes of Health that acts as a central services organization for personal property, logistics and acquisition services. 